\title{
Soziale Arbeit in Neuland
}

\author{
Soziale und politische Herausforderungen einer Sozialen Arbeit \\ in der digitalisierten Gesellschaft
}

Wenn man Angela Merkels Satz von der Digitalisierung als „Neuland" historisch einordnet, kann man zu überraschenden Schlussfolgerungen kommen. Ist der inhaltliche und methodische Stand der Sozialen Arbeit in der digitalisierten Gesellschaft vergleichbar mit dem der Armenfürsorge zu Beginn der Industrialisierung?

$\mathrm{P}$ olitiker_innen können sich nicht aussuchen, was von ihrer Arbeit bleibt. Bestenfalls sind es visionäre Begriffe wie Roosevelts „New Deal“ oder Egon Bahrs „Wandel durch Annäherung“. Schlimmstenfalls bleiben Peinlichkeiten über den Sex mit einer Praktikantin oder Running Gags, wie die berühmte NeulandÄußerung von Bundeskanzlerin Angela Merkel (2013): „Das Internet ist für uns alle Neuland. “Wenngleich diese Äußerung in Presse und IT-affinen Kreisen für Unmengen an Häme und Spott gesorgt hat, ist doch ihr Überleben ein Indikator für eine Bedeutung jenseits tagespolitischer Hektik. Hätte Otto von Bismarck bei der Einführung der Sozialversicherungen in den 1880er Jahren gesagt: „Die Industrialisierung ist für uns alle Neuland“ - er hätte mindestens die gleiche Häme informierter Kreise geerntet wie Frau Merkel. Immerhin tobte die industrielle Revolution schon mehrere Jahrzehnte durch Deutschland und Europa. Aus heutiger Sicht wissen wir, dass es, ob der Entwicklungen, die 1880 noch vor allen lagen, ein visionärer Satz gewesen wäre.

Die Soziale Arbeit, als umfassender Reparaturbetrieb für die zentrifugalen Folgen der Industrialisierung, entwickelte sich mit ihr und stets nachgängig. Erst nach

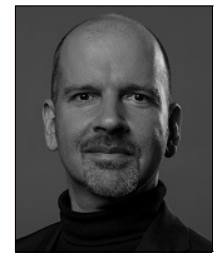

\section{Sascha Weber}

Hochschule Magdeburg-Stendal, Magdeburg, Deutschland

*1970, Dr. phil., Professor für Soziale Arbeit mit dem

Schwerpunkt Organisation und Verwaltung an der Hochschule

Magdeburg-Stendal.

Sascha.weber@h2.de

Zusammenfassung Der Beitrag skizziert anhand der Beispiele Aufmerksamkeitsökonomie und Proprietarität, dass die Digitalisierung in ihren gesellschaftlichen Auswirkungen mit der Industrialisierung vergleichbar sein könnte. Daraus folgen soziale und politische Herausforderungen an die Soziale Arbeit und die Frage nach ihrem Mandat im virtuellen Raum.

Schlüsselwörter Soziale Arbeit, Digitalisierung, Aufmerksamkeitsökonomie, Proprietarität, Mandat, Digitale Bürgerrechte und nach zeigen sich, wie seinerzeit in der Industrialisierung, die Folgen der Digitalisierung. Immerhin lagen zwischen Newcomens Dampfmaschine 1712 und der Gründung des Instituts für Gemeinwohl 1890 in Frankfurt doch ganze 178 Jahre. Ganz so viele werden es bei der Digitalisierung nicht werden. Wenn sich also 30 Jahre nach Entwicklung des World Wide Web und der dadurch möglichen Technologien die Frage nach den Folgen für die Soziale Arbeit auftut, so ist dies kein Hinweis auf eine gewisse Grundsomnolenz der Profession, sondern auf ihre normale Eigenschaft als problembearbeitende Profession, die erst auf den sozialpolitischen Plan tritt, wenn entsprechende Fehlentwicklungen, Exklusionsprozesse u. ä. gesellschaftlich präsent und ihre Nichtbeachtung mit hohen politischen Kosten verbunden ist. Dieser Beitrag versucht exemplarisch, soziale (Aufmerksamkeit) und politische (Proprietarisierung) Entwicklungen der Digitalisierung auf ihre Herausforderungen für die Soziale Arbeit hin zu befragen.

\section{Die Perfektionierung}

\section{der Aufmerksamkeitsmaschine}

Der Mensch ist als soziales Wesen auf Kommunikation und damit auf die Aufmerksamkeit seiner Mitmenschen angewiesen. Wechselseitige Aufmerksamkeit bildet die Grundlage von Erziehung, Sozialität, Kooperation. Aufmerksamkeit ist ein Treibstoff menschlicher Gesellschaft. Dennoch ist die Geschichte der Erforschung ihrer politischen und ökonomischen Nutzung noch relativ jung. Die Entwicklung planvoller „Public Relations“ (seinerzeit auch „Propaganda“) durch Edward Bernays griff auf die Psychoanalyse zurück und bildete die Blaupause für Joseph Goebbels' Kriegs- und Rassenpropaganda (Schnee 2009). Die Entwicklung von Massenmedien (von der Bibel bis heute) ermöglichte die Steuerung von Aufmerksamkeit, die zuvor nur im sozialen Nahraum entstand und verfügbar war. Aufmerksamkeit war und ist Macht- und Herrschaftsinstrument.

Mit den Massenmedien nimmt der Mensch Dinge außerhalb seiner sinnlich erfahrbaren Umwelt wahr. Die 


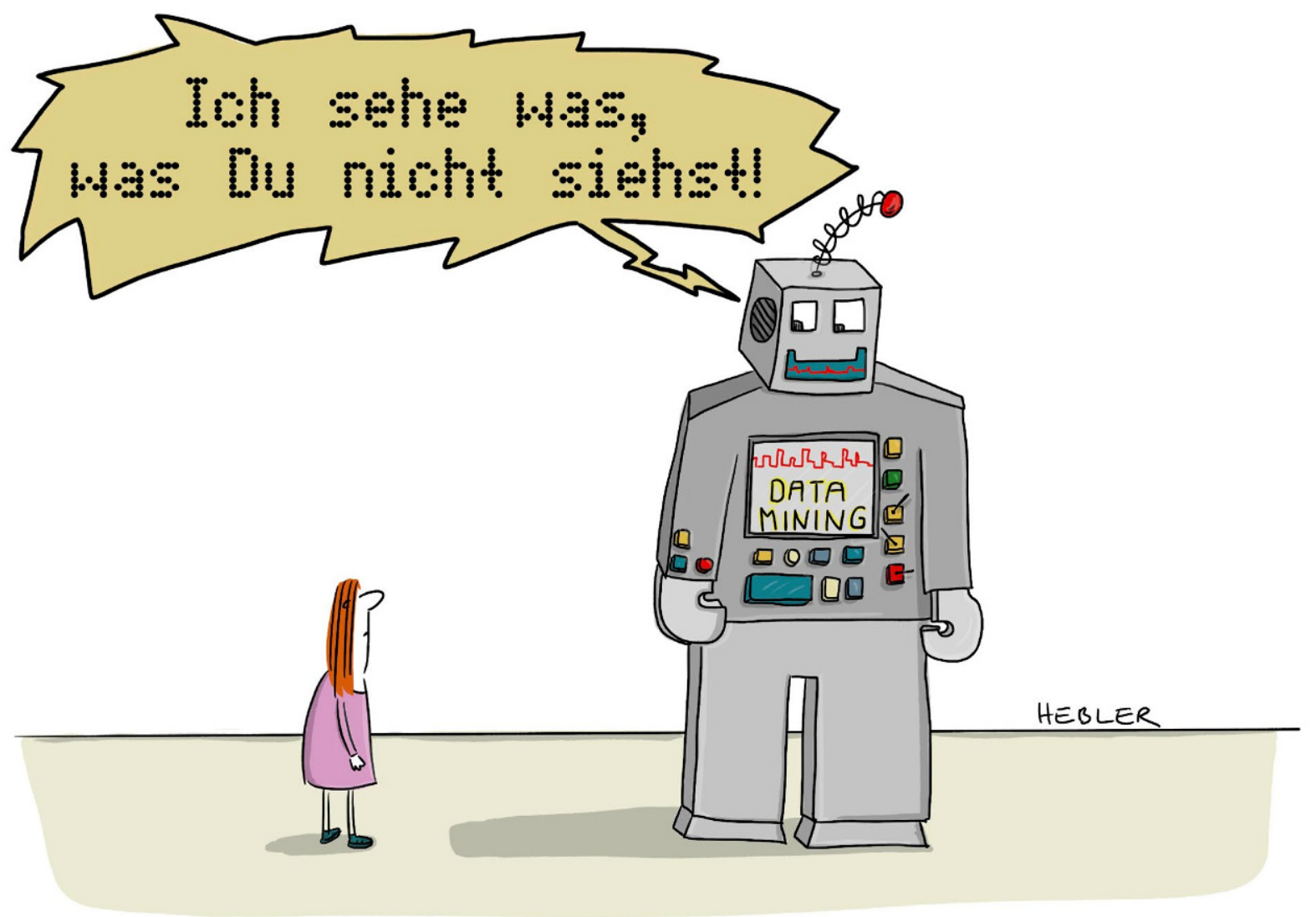

(c) Ruth Hebler

Mechanismen seiner Wahrnehmung ändern sich jedoch nicht. Menschen reagieren emphatisch auf den Schmerz Anderer. Sie teilen Angst, Erregung oder Freude. Massenmedien ermöglichen kollektive Freude über den Gewinn einer Fußball-Weltmeisterschaft und sie ermöglichen Pogrome, wie den Massenmord der Hutu an den Tutsi 1994 in Ruanda. Das Medium ist weder gut noch schlecht - es erweitert den sozialen Nahraum.

\section{Die schleichende Kommodifizierung} der Aufmerksamkeit

Schon immer war öffentliche Aufmerksamkeit ein knappes Gut, das wenigen Berufsgruppen vorbehalten war. Allen voran Geistliche leben von der Aufmerksamkeit der Gläubigen. Aufmerksamkeit wurde zunehmend notwendige Bedingung von Berufen wie Politiker, Künstler, Schriftsteller. Sie leben quasi von Aufmerksamkeit, insbesondere seit der Entstehung von Massenmedien.
Mit der Entwicklung des Internets erlebt die Aufmerksamkeit einen weiteren Wandel. Nicht mehr nur, dass praktisch jeder Mensch an jedem Ort über jede Information verfügen kann - quasi die Empfänger-Eigenschaft sich universalisiert. Auch die Sender-Eigenschaft wird popularisiert. Die Sender-Eigenschaft ist nicht mehr Privileg von Zeitungen, Literaten, Rundfunkanstalten oder Wissenschaftlern. Jeder kann senden und sich damit das knappe Gut Aufmerksamkeit beschaffen. Jedes Like, das auf ein Posting in soziale Netzwerke folgt, wird zum kleinen Dopamin-Kick fürs Belohnungssystem. Soziale Netzwerke werden darauf hin optimiert, ihre Nutzenden möglichst oft zum Posten und Liken $\mathrm{zu}$ animieren, und sind ebenfalls darauf optimiert, diese Tätigkeit in (Werbe-) Einnahmen zu transformieren. Diese Demokratisierung von Senden und Empfangen kann positiv sein, wenn es sich um Information handelt; sie zeigt ihre dunkle Seite, wenn der Brandbeschleuniger der Aufmerksamkeit ins Spiel kommt: Die Emoti- 
on. Das FOMO (Fear-Of-Missing-Out)-Syndrom, was Handybesitzer auf jedes Piepen ihres Gerätes mit Nervosität reagieren lässt, dokumentiert die Spannung aus Lust und Angst der Nutzenden. Trolle genießen die Aufmerksamkeit, die ihnen in den Foren des Internets gezollt wird und die Seitenbetreibenden profitieren wiederum an jedem Shitstorm. Verschwörungsmystiker_innen und twitternde Lügen-Präsidenten nutzen die gleichen Mechanismen und werden mit Aufmerksamkeit (likes, views, re-tweets ...) belohnt. Georg Franck (2007) bezeichnet dieses Geschäftsmodell bereits 1998 treffend als „Ökonomie der Aufmerksamkeit“.

Ganz en passant, ganz ohne Weltverschwörung, entsteht durch die Demokratisierung von Senden und Empfangen ein Neuland, in dem die Lautesten und Emotionalisierndsten die Aufmerksamkeit erhalten. Rationale Argumentation und wissenschaftliche Expertise, demokratische Debattenkultur und gemeinsame Grundwerte werden darin $\mathrm{zu}$ „alternative facts“ relativiert. $\mathrm{Zu}$ sammen mit der umfassendsten Datensammlung über jede_n Nutzer_in der Geschichte, ist der Tisch für ein autoritäres Regime bestens bereitet.

Ähnlich wie die Industrialisierung fördert die Digitalisierung eine Unmenge von Innovationen, die durch sie möglich werden. Und in ähnlicher Weise werden die negativen Innovationen sukzessive gesetzlich reguliert werden müssen. Die Industrialisierung erzeugte mit den gleichen Mitteln Antibiotika und sauberes Trinkwasser, wie sie Heroin und chemische Kampfstoffe produzierte.

Die Digitalisierung erzeugt z. B. kommunikative Inklusion für Menschen, die bislang in ihrer Lebenslage isoliert und gesellschaftlich exkludiert waren oder aufgrund von Beeinträchtigungen nicht am gesellschaftlichen Diskurs teilnehmen konnten. Sie gibt allerdings auch Wirrköpfen die Möglichkeit zur Verbreitung von Ideen, die besser nicht den Kopf verlassen hätten und erlaubt ihnen, mitsamt ihren Followern die Axt an die Grundwerte der Gesellschaft zu legen.

\section{Wiederkehr der Arbeiterkolonien}

Service-Providern, wie z. B. Sozialen Medien, gelingt es, die Kosten des Anbieterwechsels (switching costs) so in die Höhe zu treiben, dass ihre Nutzenden sich das selbstgewählte Apple oder Facebook-Biotop notgedrungen schönreden. So ging es schon den Arbeiterfamilien zu Beginn der Industrialisierung, die bei den KruppStahlwerken in Essen arbeiteten, in Krupp-Wohnungen lebten und ihren Lohn in Krupp-Magazinen und KruppBierhallen wieder ausgaben und von denen Loyalität gegenüber der Firma erwartet wurde (Krämer 2010). Damals wie heute bestimmt der Eigentümer.
Während man die im Zuge der Industrialisierung entstandene Trennung von Wohnen und Arbeiten und die daraus resultierenden Probleme als zentrale Herausforderung für die entstehende professionelle Soziale Arbeit im 19. und 20. Jahrhundert betrachten konnte, könnte dies die Trennung von physischer und virtueller Existenz im Zuge der Digitalisierung im 21. Jahrhundert werden. Die virtuelle Existenz meint damit nicht eine Science-Fiction-Parallelwelt mit bunten Avataren, sondern die ganz normale zweite Ebene, die einen zunehmenden Teil unseres Alltags darstellt. Als Kund_in von Online-Shops, Streaming-Diensten, Messaging-Apps, Video-Konferenzteilnehmende, Nutzende und Coproduzierende von Sozialen Netzwerken. Diese zweite Welt hat die Welt und das Geschäftsmodell der Krupp'schen Arbeiterkolonien in Gestalt der Proprietarisierung wiederbelebt. Diese Welt hat immer eine_n Eigentümer_in, der/die die Regeln bestimmen darf. Diese_r Eigentümer_ in verkauft keine Güter mehr, er/sie verkauft Lizenzen für die Nutzung eines Dienstes, die auch weitgehende Rechte an den von Nutzenden bereitgestellten Daten umfassen. Im Gegenzug hat der/die Nutzer_in in der Regel keinen Einblick in Algorithmen und Weiterverwendungen. Mit dem Arbeiter der Krupp'schen Kolonie hat der Nutzende des proprietären Internets gemein, dass er kaum Rechte hat und die wenigen, über die er verfügt, kaum durchsetzen kann.

\section{Welche Folgen hat die Proprietarisierung?}

Die virtuelle Welt ist proprietär. Obwohl es kaum einen offeneren Raum als das Internet gibt, ist das Internet kein öffentlicher Raum und erst recht nicht demokratisch konstituiert. Es gehört nicht dem Gemeinwesen oder einem Staat, die rechtliche Grundannahme, dass Recht an einem „Ort“ gilt, stößt im Internet an ihre Grenzen und ist nicht klar zu beantworten. Gilt das Recht des Staates, in dem der Server steht? Oder jenes, wo der Provider seinen Sitz hat? Oder gelten die Regeln des Ortes, an dem die Interaktion stattfindet?

Das Gelten von Recht geht einher mit der Frage, welche Rechte der Nutzende eines Dienstes hat und inwieweit der/die Inhaber_in eines Accounts Träger_in von Menschen- und Bürgerrechten ist oder sein kann?

Aus Sicht der Sozialen Arbeit wäre zu fragen, welche die Bürgerrechte im Internet sind? In einem proprietären Internet macht der Eigentümer die Regeln. Facebook, Twitter u. a. können praktisch täglich ihre Nutzungsbedingungen ändern, ihren Geschäftssitz verlagern, Daten verkaufen, verändern oder vernichten. Sie können Nutzer aufnehmen und ausschließen. Das Akzeptieren von geänderten Nutzungsbedingungen durch die Nutzer_innen wird zur Farce. Wenn die Nutzung eines Dienstes 
zum Grundrecht (vgl. die Rechtsprechung des BVerfG zum Telefonanschluss) wird, weil der Ausschluss davon zum „sozialen Tod“ des Nutzenden führen würde, wo sind dann die Grenzen des Dienstanbieters?

Wenn das Smartphone zur Grundausstattung des/der Bürger_in gehört, um einen Behördentermin zu vereinbaren, dann hört Apple oder Google stets mit, wie es um die Arbeitslosigkeit des Nutzenden bestellt ist. Er/ sie kann sich auch nicht mehr entziehen, wenn im ländlichen Raum die Versorgung mit Konsumgütern nur mehr über den Online-Handel möglich ist, weil der stationäre Handel längst geschlossen wurde. Diese Beispiele sind Hinweise darauf, dass sich die Tore der Arbeiterkolonien langsam wieder schließen.

\section{(K)ein Mandat in Neuland}

Die Politik steht durch die Digitalisierung unter dem Handlungsdruck, neben den realen auch virtuelle Bürgerrechte zu ermöglichen. In der Industrialisierung war dies die Einrichtung von staatlicher Aufsicht (z. B. Arbeiterrechte, Gesundheit und Jugendhilfe). Heute steht die Politik vor den Schranken der Arbeiterkolonien, deren Pförtner proklamieren, jeder sei freiwillig hier und hätte am EULA (End-User-Licence-Agreement) den JaButton geklickt. Die neuen Krupps propagieren sogar einen „Contract for the web“ (https://contractfortheweb.org/), in dem sie die gegenwärtigen GovernanceStrukturen des Webs als erstrebens- und erhaltenswert für Government, Companies und Citizens beschreiben. Soziale Arbeit tritt in der Regel politisch legitimiert auf den Plan, wenn die Grenzen der Steuerungsmittel Geld und Recht ausgeschöpft sind. Sie braucht ein Mandat und ein Ziel für ihre Intervention. Bislang hat die Soziale Arbeit nur an den Grenzen von Neuland ein Mandat, wo sich Realität und Virtualität überschneiden. Es ist also im Folgenden zu diskutieren, wie das Mandat der Sozialen Arbeit aussehen könnte und wie es in einer proprietären Umgebung durchsetzbar wäre. Daran und danach schließen sich Fragen nach der Methodik Sozialer Arbeit in der virtuellen Welt und Fragen nach der Kompetenz von Sozialarbeiter_innen in diesem neuen Feld.

\section{Data Mining im Bereich Soziale Arbeit}

Die Forschung über Soziale Arbeit muss die Wahrnehmung dieses Mandats kritisch begleiten. Gleichzeitig muss sich die Soziale Arbeit Kompetenzen und Handlungsformen aneignen, mit denen sie in der Lage ist, in einer virtuellen Welt wirksam zu intervenieren.

In den Beiträgen von Tilman Kloss und Konstantin Kirchheim ist zu erkennen, welche Möglichkeiten sich aus der Verfügbarmachung der Daten über die Soziale Arbeit und der Nutzung der softwaregestützten
Auswertung großer Datensätze erschließen lassen. Die interdisziplinäre Zusammenarbeit zeigt zudem, dass die Bezugswissenschaft Informatik mehr methodische Schnittstellen vorweist, als der Einsatz von PCs in Jugend- und Sozialämtern nahelegt.

Funding. Open Access funding provided by Projekt DEAL.

Open Access. Dieser Artikel wird unter der Creative Commons Namensnennung 4.0 International Lizenz veröffentlicht, welche die Nutzung, Vervielfältigung, Bearbeitung, Verbreitung und Wiedergabe in jeglichem Medium und Format erlaubt, sofern Sie den/die ursprünglichen Autor(en) und die Quelle ordnungsgemäß nennen, einen Link zur Creative Commons Lizenz beifügen und angeben, ob Änderungen vorgenommen wurden.

Die in diesem Artikel enthaltenen Bilder und sonstiges Drittmaterial unterliegen ebenfalls der genannten Creative Commons Lizenz, sofern sich aus der Abbildungslegende nichts anderes ergibt. Sofern das betreffende Material nicht unter der genannten Creative Commons Lizenz steht und die betreffende Handlung nicht nach gesetzlichen Vorschriften erlaubt ist, ist für die oben aufgeführten Weiterverwendungen des Materials die Einwilligung des jeweiligen Rechteinhabers einzuholen.

Weitere Details zur Lizenz entnehmen Sie bitte der Lizenzinformation auf http://creativecommons.org/licenses/by/4.0/deed.de

\section{Literatur}

Franck, G. (2007). Ökonomie der Aufmerksamkeit. Ein Entwurf. München: DTV.

Krämer, S. (2010). Deutsche Unternehmer und ihre Arbeiterkolonien im 19. und frühen 20. Jahrhundert. Kunstgeschichte. Open Peer Reviewed Journal (urn:nbn:de:0009-23-26477).

Schnee, P. (2009). PR-Erfinder Bernays: Der Überzeugungstäter. In: DER SPIEGEL, 30.09.2009. https://www.spiegel.de/geschichte/pr-erfinder-bernays-a-948512.html. Zugegriffen: 2. Aug. 2020. 